\title{
The (bi)sexual brain
}

\author{
SSS Science \& Society Series on Sex and Science
}

\section{David Crews}

M ost animals begin their lives as a single cell, grow and develop into one of two sexes and, when mature, interact with the other sex to conceive a new single cell that starts the cycle anew. This basic equation underlies all aspects of sexuality; that is, specific traits evolved to attract members of the opposite sex, ensure copulation and thereby reproduction. It also suggests that all life forms evolved from ancestors that could adopt both male and female roles (Fig 1) bisexual life forms-and we can find elements of bisexuality throughout all life processes, including the brain functions in higher animals that control sexual behaviour. But, semantics can manipulate perception: the general view still defines male and female in regard to the opposite sex and focuses on differences rather than similarities. However, to explain sexual behaviour, there is a new perspective that regards it as an emergent property of specific areas of the brain.

\section{Initially, humans and animals were regarded as innately bisexual: an individual's sexuality could be placed on a continuum between masculinity and femininity}

How the brain controls sexual behaviour is the topic of an interdisciplinary science that is rooted in ancient myths and scriptures [1,2]. Initially, humans and animals were regarded as innately bisexual: an individual's sexuality could be placed on a continuum between masculinity and femininity. Two centuries ago, the Viennese psychiatrists Richard von KrafftEbing and Sigmund Freud, and the German otolaryngologist Wilhelm Fleiss, posited that the mechanisms controlling sexual behaviours were equally represented in specific 'centres' in the brain, and that a dynamic tension between these centres accounts for the degree of masculinity or femininity. Their view was based on the discovery that, during embryogenesis, the ovaries and testes developed from a common anlagen (precursor), whereas the accessory sex structures developed from different anlagen [3,4].

This perspective eventually faded away until, about 60 years ago, a new paradigm emerged according to which males and females have fundamentally different brains. Unfortunately, this was a classic example of throwing the baby out with the bathwater, as the new paradigm has not helped to explain sexual behaviour in higher animals. A new perspective that incorporates elements of both previous ones has gained traction: that sexual behaviour is an emergent property of a common network of brain nuclei and functions to ensure that sexual partners show complementary behaviours. This new hypothesis is consistent with findings on the genetics of sex determination and sexual differentiation, the evolution of transcription factors and the central nature of complementarity in life processes.

B isexuality is in fact an integral part of all organisms and is grounded in the very origin of sex, among single cells and simple multicellular organisms that evolved specialized cells for reproduction: the gametes. The evolution of gametes as containers of heritable material coincided with the development of meiosis, such that gametes must combine to create a new organism. Initially, there were no differences between male and

female individuals; similarly, male and female gametes were of equal size (isogamy). Eventually, the gametes began to differ in size in most lineages (anisogamy). Those animals that produce small gametes (sperm) are usually called males, whilst those that produce larger gametes (eggs) are called females. This semantic dichotomy, although necessary, has shaped how we view all things sexual; that is, as elements more separate than alike.

\section{Bisexuality is in fact an integral part of all organisms and is grounded in the very origin of sex...}

Most organisms are sexual but not necessarily gonochoristic; that is, separate sexes in separate individuals. For example, various forms of hermaphroditism are capable of self-fertilization, but this is rare when they have an opportunity to copulate with other hermaphrodites. Nonetheless, gonochorism is the norm in animals. Although asexuality and parthenogenesis_cloning or 'virgin' birth-exists, such modes of reproduction did not evolve originally, but derived from sexual reproduction.

SSS

\section{Science \& Society Series on} Sex and Science

Sex is the greatest invention of all time: not only has sexual reproduction facilitated the evolution of higher life forms, it has had a profound influence on human history, culture and society. This series explores our attempts to understand the influence of sex in the natural world, and the biological, medical and cultural aspects of sexual reproduction, gender and sexual pleasure. 


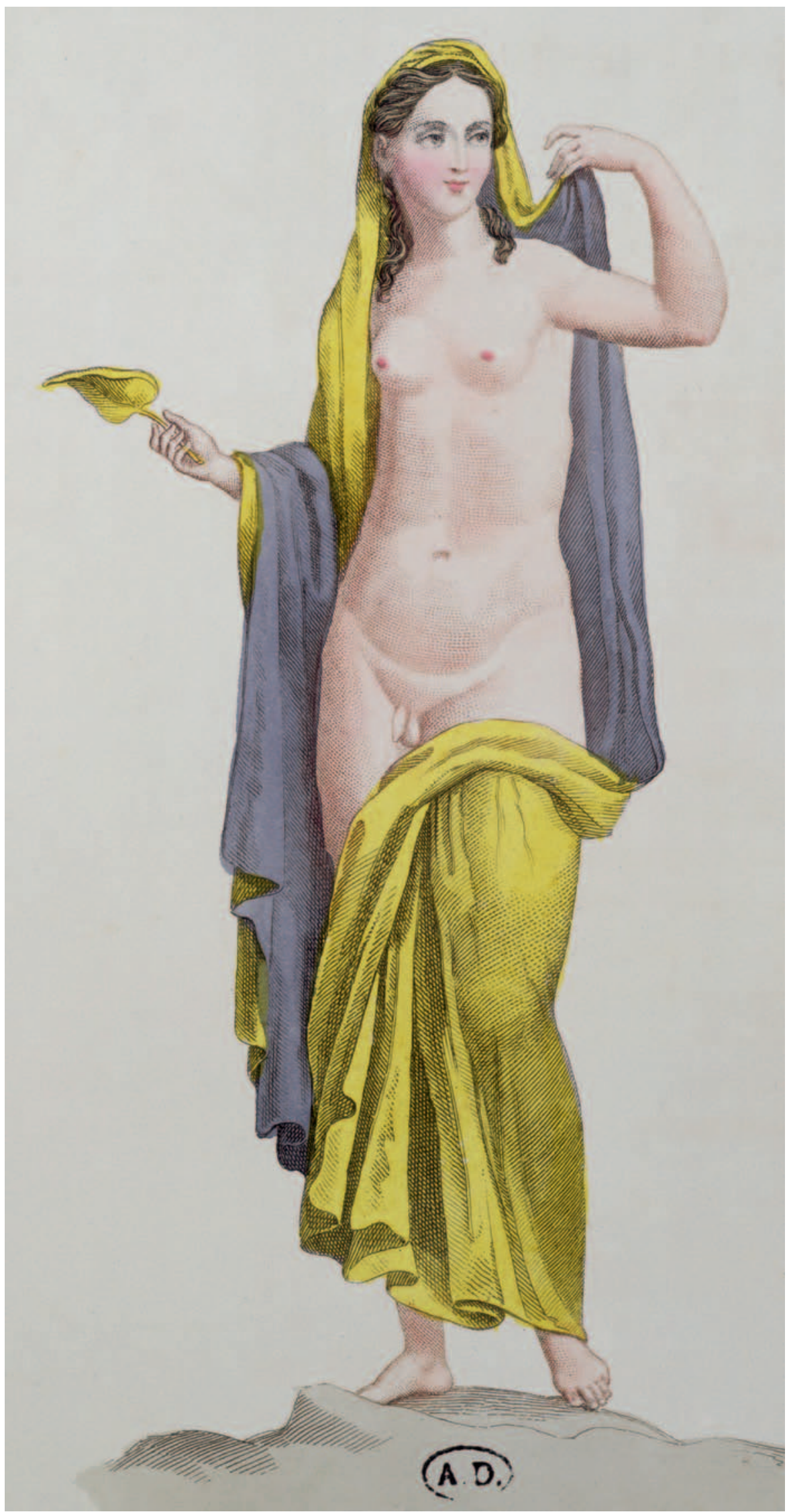

4 Fig 1 Hermaphrodite, from a Pompeian fresco, c.1800 (coloured engraving), French School (nineteenth century)/Bibliothèque des Arts Decoratifs, Paris, France/Archives Charmet/ The Bridgeman Art Library.

In 1905, Nettie Stevens discovered sex chromosomes and their role in sex determination. In a few decades it became generally accepted that, in animals and certainly all vertebrates, the sex of the offspring is determined at the moment of fertilization by complementary heritable units of genetic material. By mid-century, it was established that genetic control of sex determination in turn drives the differentiation of the sexes in regard to physiology and behaviour. However, further research showed that some amphibians, many fish and turtles, and all crocodilians depend on external environmental cues to determine sex. Moreover, in some fish, this process can persist throughout life, as these animals can switch sex in adulthood. Research has also revealed that both environmental and genomic triggers of sex determination can work simultaneously in some species.

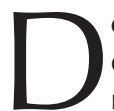
espite this diversity in sexdetermining mechanisms, the molecular basis is rather similar: a constellation of evolutionarily conserved genes-not necessarily on the sex chromosomes-orchestrate whether testes or ovaries will be formed from the genital ridge. Thus, even species without sex chromosomes still develop into males and females in response to environmental cues. Whatever the switch or trigger, it engages a primary gene 'cassette' of evolutionarily conserved and functionally related genes that interact to determine the fate of the gonads. The nature of these interactions changes through development and this cassette engages other cassettes of integrated gene assemblies, such as those responsible for the differentiation of secondary and accessory sex structures.

Thus, the developmental decision of male versus female does not flow through a single gene, but is instead determined by a parliamentary system involving networks of genes. All systems with different degrees of inherited and environmental influences could operate this way, merely by varying the inputs to the networks. The binary nature of sex determination in turn led to the evolution of distinct developmental trajectories 
that generate complementary morphological, physiological and behavioural differences between the sexes, which facilitate their ultimate union for reproduction.

Sexual differentiation requires signalling systems that encourage alternative developmental pathways. The agents of these changes are hormones, principally the sex steroid hormones. They are not involved in determination of gonad type in mammals and birds. However, in all cold-blooded vertebrates there is a near universal role of steroids and their enzymes in overriding the primary cassette of sex-determining genes to redirect the fate of the gonads.

L ikewise, both sex hormones and genetic networks determine the sexspecific behaviour of males and females. As different gametes are required to conceive a new organism, different sex-specific behaviour is important for achieving copulation and reproduction. This behavioural interaction between male and female is a relationship, and a useful way to consider relationships is in terms of stimulus-response complementarity, as defined by the American psychologist Frank Beach [5,6]. At its most basic level, and this sounds deceptively simple and obvious, for a male to successfully mate, the female must be receptive to his efforts and vice versa.

\section{...the developmental decision of male compared with female does not flow through a single gene, but is instead determined by a parliamentary system involving networks of genes}

The functional consequence of this complementarity in mating behaviour is that the male provides the behavioural stimuli necessary to ensure normal ovarian activity in the female. This behavioural facilitation of reproductive function is reciprocal. That is, the female is crucial to maximizing the male's fertility, just as the male is important to the female's reproductive activity. Thus, if each individual is allowed to choose its sexual partner, and if that choice is reciprocated or mutually agreed upon, fecundity and reproductive success is increased relative to when choice is forced [7].

Behavioural facilitation of reproduction has been described in all life forms, even in unicellular organisms that have mating types rather than different sexes as in multicellular organisms, and in plants. Parthenogenetic fruit flies and whiptail lizards - there are no males in these species-also show a behavioural facilitation and synchronization of reproduction. The fact that it occurs in all kinds of organisms, and that the diversity of organisms exhibiting behavioural facilitation is greater than the diversity of organisms that sexually reproduce, suggests that facilitation is more ancient than sexual reproduction. The original purpose seems to be synchronization of reproduction in a population so that young are produced at the same time, which minimizes the probability of an individual's young being eaten by predatorsthe advantage is the same for gonochoristic and cloning species.

There is still widespread belief that males and females are fundamentally different because of their difference in genetic constitution. And there is evidence that the presence of heterogametic sex chromosomes in mammals, and their dosage in birds, also affects brain development and function. These and other genes are probably involved in sensory decoding of the sexual stimuli crucial to mate choice, but the extent to which they are important in an evolutionary sense is debatable. There is no argument that the main sex differences in the brain are shaped by the sex hormones. This brings us back to the point that basic, conserved mechanisms underlie sex determination and sexual differentiation including processes in the brain, which contains a conserved neural network of nuclei that regulate sexual behaviours.

$\mathrm{t}$ also means that this neural network must form and organize early in development, so that adult animals are able to show sex-specific and complementary behaviour that is necessary for copulation and reproduction. The origins of this concept result from a mistaken analogy with the differentiation of genital tracts [8]. Both would take place during a limited time early in fetal development, both would be irreversible and both would generate separate organs or neural structures that enable copulation and mediate sex-specific behaviour. The first two have been confirmed in extensive research with rodents, revealing a male-specific testosterone surge towards the end of in utero development that enables later expression of male behaviour (masculinization), whilst disabling later expression of female behaviour (defeminization) [9]. The last element however has not stood up to experimental testing. Instead, a new perspective considers the network of limbic and hypothalamic nuclei involved in the control of sexual behaviour as a single entity, organized in a male-typical or female-typical way. The implication of this perspective is that it is contradictory to the independent existence of separate 'centres' for male- and female-typical sexual behaviour.

An influential conceptualization of how the brain might differentiate in males and females was the orthogonal model drawn up by Richard Whalen [10]. He proposed that sexuality is not a linear scale with male and female occupying opposite ends; instead, sexuality involves two separate dimensions that determine the degree of masculinization and feminization, respectively. During development, these are organized differently by gene and hormonal activity to generate the behaviour typical of each sex [13].

\section{...rather than enabling males and females to do something that the other sex cannot, sex differences enable males and females to be better at the things they do}

Early studies already identified these entities as separate areas of the brain: male behaviour-mounting females-involves the medial preoptic area (POA), whereas receptivity-typical of females-is controlled by the ventromedial nucleus of the hypothalamus $(\mathrm{VMN})$. According to Whalen's orthogonal model, these nuclei would be the final steps in dual neuronal circuits, which are differentially influenced by the hormonal milieu during early development. However, extensive research into the mammalian POA and VMN could not prove that the differences seen are not simply correlates of observed behaviours. As Per Södersten put it, "the search for morphological sex differences in adult rat brains that are caused by the 'organizing effect of perinatal androgen' and that can be related to sex differences in behaviour has not been fruitful and may continue unrewarded" [11]. 

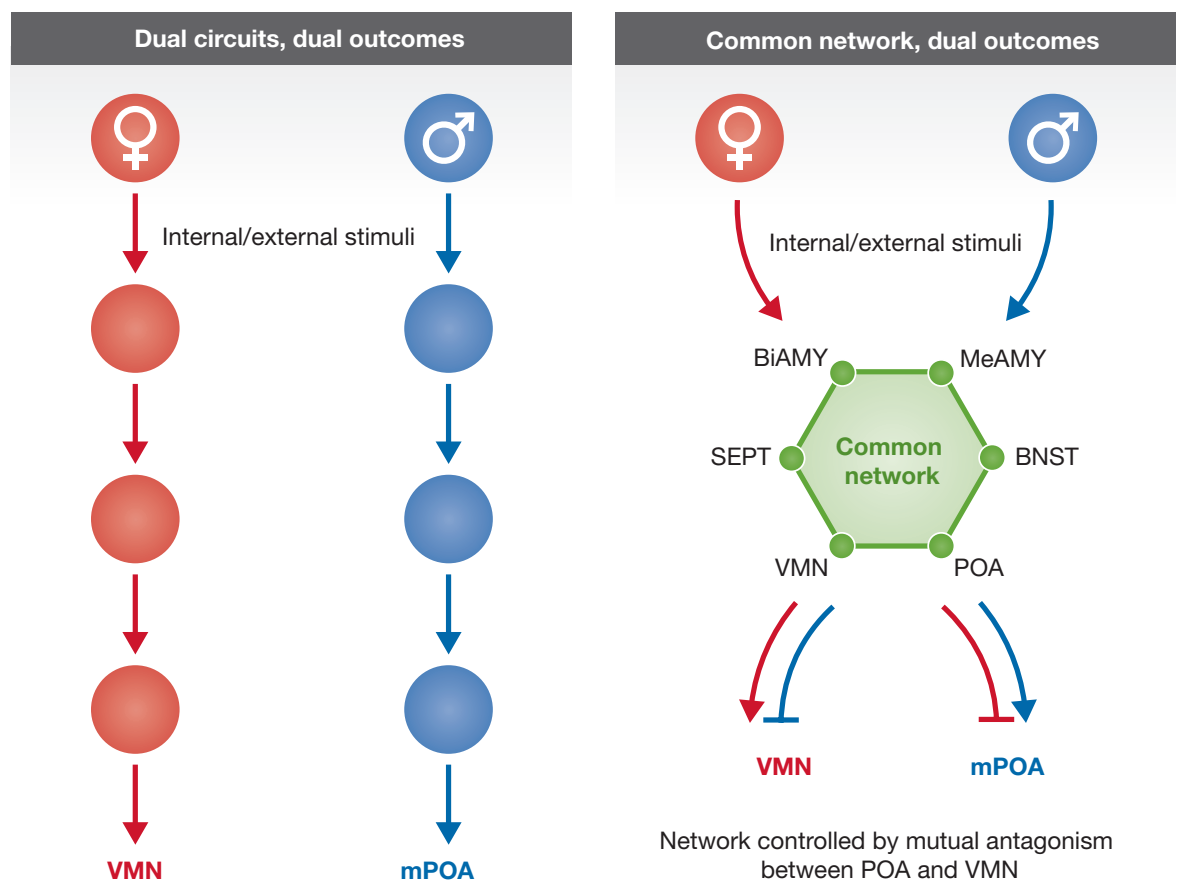

Fig 2 | Dual circuit model compared with common network model. The dual circuit model, after Whalen's model concept, suggests that sex hormones act early in life to induce (or not) the development of independent neural circuits subserving male- and female-specific sexual behaviours. These behaviours each have their own final integrative centres: the VMN in females and the POA in males. In the common network, both behaviours can be expressed depending on external stimuli and complementary activityactivation or deactivation - in the VMN or POA. BiAMY, basolateral amygdala; BNST, bed nucleus of the stria terminalis; MeAMY, medial amygdala; (m)POA, medial preoptic area; POA, preoptic area; SEPT, septum; VMN, ventromedial nucleus.

$\mathrm{T}$ he question, then, is whether there are dual circuits or a single network with two distinct foci that create alternative outputs (Fig 2). It is obvious that sexual behaviour is the result of multiple brain nuclei acting in concert, in addition to external stimuli and the hormonal history of the participating individuals. Sarah Newman proposed an interpretation of this work as a social behaviour network [12]. By shifting the focus of study from single nuclei (node) in isolation to integrated networks-multiple nodes of brain nuclei that are interconnectedNewman predicted that this would lead to new insights into brain-behaviour relationships. Important for this discussion, this hypothetical model only focused on sex differences and did not consider possible interactions in the network when animals display heterotypical sexual behaviours. Using her work as a platform, I have applied it to empirically derived data in several animal systems to show how both heterotypical and homotypical behaviour results from network activity, and how both are ultimately determined by the reciprocally inhibitory interaction of two root nodes: POA and VMN [13].

So far, research to support the dual circuits model has focused on studying the sex-typical behaviours - mounting in male individuals, receptivity in female individugenerated independent models of sexspecific behaviour that do not consider the partner's sex. By contrast, the common network model describes how mutually from the action of hormones in a single neural network. This also reflects our growing knowledge about the flexibility and adaptability of the brain, and the tight connections between functional centres or nuclei that determine which of a range of mutually exclusive outcomes is appropriate for response to environmental cues. Building on this insight, the common network model suggests that sex-specific behaviours are generated by specific patterns of neurotransmitters, and other molecular interactions, in two functionally associated nuclei [13]. als-in isolation. Not surprisingly, it has exclusive behaviours can be generated
$\mathrm{T}$ here is ample evidence that the POA and VMN are involved in the control of male-typical and female-typical sexual behaviour, respectively. What is less accepted, however, is the possibility that the two nuclei work in concert, albeit in a mutually antagonistic way, despite significant findings that suggest reciprocal inhibition between the two. Injecting testosterone into the VMN of castrated male rats restores sexual motivation but not copulation, whereas the addition of androgen receptor antagonists or lesions within the VMN impairs both behaviours. POA and VMN also have opposing roles in the control of female reproductive behaviour such that the "net effect of the outputs from the preoptic region is to reduce feminine-typical behaviour and to increase male-typical behaviour" [14]. Neuronal activity increases in the VMN during sexual receptivity in the female rat, and is reduced when there is increased activity in the POA. The effects of different excitatory and inhibitory amino acid neurotransmitters are opposite in the VMN and POA. This and other evidence demonstrates how $\mathrm{POA}$ and $\mathrm{VMN}$ are functionally related and mutually oppressive.

A model that considers POA and VMN as independent anatomical units, one of which determines the animal's sexual behaviour, is therefore not satisfactory, nor is it supported by evidence. The old model should be rejected in favour of a mutual inhibition model in which these nodes of a common network work actively together, yet antagonistically in individuals of both sexes during courtship and mating to ensure behavioural complementarity.

$\mathrm{T}$ he central issue in science is the proof or disproof of a hypothesis. However, it is rather difficult to prove which of the two models-a common network leading to different outcomes, or the domination of one of two neural circuits depending on the sex-is correct. Indeed, any species with heritable sex chromosomes is not suitable for investigating this particular question, as the sexes in such species differ in a few crucial genes and, consequently, have developed in different hormonal milieus and with different life histories.

Fortunately, we can look to nature for another model with which to test the hypothesis. The first problem of sex chromosomes is easily dealt with as many species lack such heritable units, yet develop as either male or female. Many reptiles show 

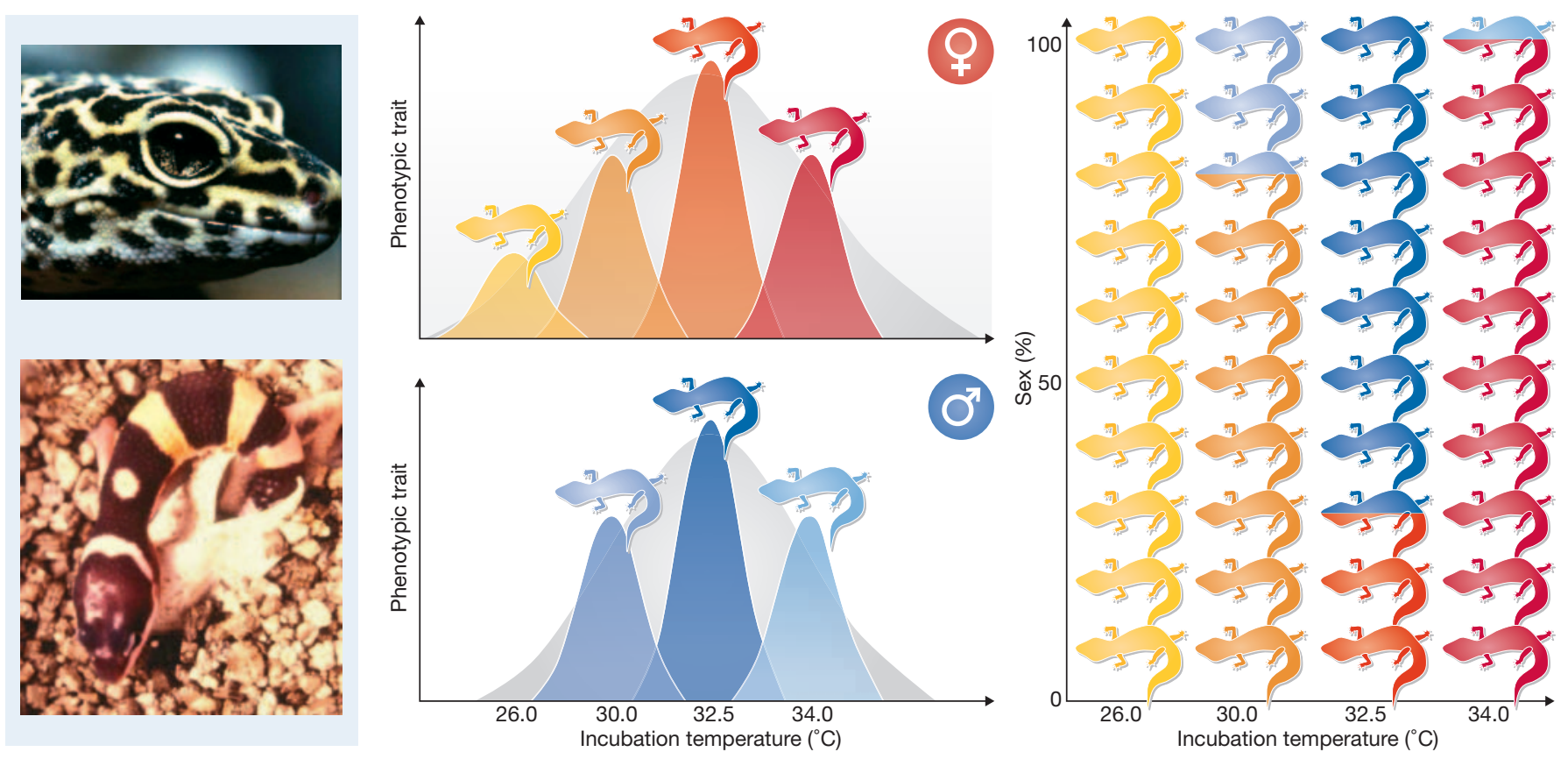

Fig 3 In many reptiles, the incubation temperature of the developing egg determines different phenotypic traits of the adult individual, including sex. In the leopard gecko, an incubation temperature of $26^{\circ} \mathrm{C}$ generates only female individuals. At $30^{\circ} \mathrm{C}$ and $32^{\circ} \mathrm{C}$, the ratio of males increases to $25 \%$ and $75 \%$, respectively, and drops to $5 \%$ or lower at $34^{\circ} \mathrm{C}$.

temperature-dependent sex determination (Fig 3). Most extreme temperatures produce only one sex whilst intermediate temperatures produce a mixture of males and females with different morphological, physiological, behavioural and neural phenotypes. If we look at males and females and analyse their metabolic activity according to the social behaviour network, we find that there is little difference other than in the POA and $V M N$ nodes.

Simultaneous hermaphrodites, which produce both sperm and egg but never at the same time, are another useful model for looking for a common network characteristic. As each animal can adopt both male and female roles, and switch throughout adult life, they obviously have a bisexual brain in terms of organization and function. Indeed, Leo Demski showed that sperm release in the sea bass could be achieved by stimulating one brain area, whereas stimulation of another brain area caused egg release [15]. The social stimuli are transduced into signals that modulate neuropeptides and neurotransmitters, pituitary gonadotropins and steroid hormones. Thus, organisms that lack genetic sex determination, and develop and age in entirely different endocrine milieus, can change sex as adults depending on environmental cues and behave in a gamete-appropriate manner. This suggests that a common neural network underlies such changes.

$\mathrm{T}$ he ultimate proof of a common neural network with reciprocal inhibition between the POA and the $\mathrm{VMN}$ nodes comes from studies of the all-female lizard Cnemidophorus uniparens, a species in which all individuals are morphologically female and reproduce by obligate cloning. This animal model allows us to deconstruct the confounding properties of genotype-, sex hormone- and developmental-specificity mentioned above that plague conventional mammalian model systems.

The maternal ancestor of $C$. uniparens is Cnemidophorus inornatus, a typical gonochoristic species with male and female individuals that look and behave in a sexually dimorphic manner; that is, males mount whilst females do not, and male mounting is dependent on androgens acting on the POA. Female $C$. inornatus only show receptivity when the follicles are large and oestrogen levels high; oestrogen acts on the VMN.

Remarkably, the behaviour of individual C. uniparens are identical to both the male-typical and female-typical behaviours of their sexual congeners. This we call 'pseudosexual behaviour' and their union 'pseudocopulation'. Pairs of C. uniparens are always complementary, and their behaviour is linked to their ovarian state: the mounting individual and displaying male-like pseudosexual behaviour is generally postovulatory and has elevated progesterone levels, whereas the receptive individual is preovulatory and has high oestrogen levels.

The POA and the VMN of C. uniparens do not change in cell size or number during these different behavioural phases, although they do differ in metabolic activity. Predictably, there is high metabolic activity in the POA and low activity in the VMN during the male-like pseudocopulatory behaviour and vice versa [17]. Moreover, the experimental application of androgen or progesterone into the POA of both male C. inornatus and C. uniparens triggers mounting behaviour, but fails to produce either mounting or receptive behaviour when administered into the VMN. As expected, the application of oestrogen to the $\mathrm{VMN}$ causes receptive behaviour in female C. inornatus and C. uniparens.

Both the POA and the VMN are dimorphic in size: the POA is larger in sexually active male $C$. inornatus than in conspecific females or in the descendant parthenogenetic C. uniparens. However, this seems to be a correlation, rather than an underlying cause of male-like pseudocopulatory behaviour: neither the POA region nor somal area 
increase in C. uniparens showing male-like pseudocopulatory behaviour. Any differences in the POA between the parthenogens displaying male-like or female-like pseudosexual behaviours have been subtle ones at the levels of gene expression and neurotransmitters [13]. The parthenogenetic whiptails thus demonstrate that developmentally determined long-term differences in brain morphology are less relevant for sex-specific behaviour than the short-term activity of these nuclei, orchestrated by external stimuli and the hormonal state of the individual.

$\mathrm{n}$ his writings about the nature of the neuroendocrine control of sexual behaviour in vertebrates, Frank Beach listed four essential points: both males and females are able to display the sex-specific behaviours of the opposite sex; the brain must support these opposite behaviours; each sex is predisposed to behaving consistently with its own sex; and the stimulus animal is essential for triggering complementary behaviour [13].

Experimentally, one can further examine how males and females can behave in both a sex-appropriate manner, as well as in the manner appropriate to the opposite sex. It also begs the question of what are the functions of the observed sexual dimorphisms in brain structure, if they do not mediate sex-typical copulatory behaviour.

Taken together, this evidence suggests that modern vertebrates are fundamentally bisexual in nature; that the neural mechanisms mediating both male and female copulatory behaviour are normally inhibited; and that activation provides relief from some of the inhibitory inputs. In other words, rather than enabling males and females to do something that the other sex cannot, sex differences enable males and females to be better at the things they do [13].

\section{ACKNOWLEDGEMENTS}

To the memory of Eugen Steinach and Frank A. Beach, who had the insight, but not the organism. Parts of the text have been adapted from reference [13], with permission from Elsevier.

\section{CONFLICT OF INTEREST}

The author declares that he has no conflict of interest.

\section{REFERENCES}

1. Sengoopta C (2006) The Most Secret Quintessence of Life: Sex, Glands, and Hormones, 1850-1950. Chicago, Illinois, USA: University of Chicago Press

2. Money J (1990) Androgyne becomes bisexual in sexological theory: Plato to Freud and neuroscience. I Am Acad Psychoanal 18: 392-413

3. Rado S (1940) A critical examination of the concept of bisexuality. Psychosom Med 2: 459-467

4. Stoller RJ (1972) The 'bedrock' of masculinity and femininity: bisexuality. Arch Gen Psychiatry 26: 207-212

5. Beach FA (1976) Cross-species comparisons and the human heritage. In Human Sexuality in Four Perspectives (eds Beach FA, Diamond M), pp 469-486. Baltimore, Maryland, USA: Johns Hopkins University Press

6. Beach FA (1979) Animal models for human sexuality. In Ciba Foundation Symposium 62: Sex, Hormones and Behavior (eds Porter R, Whelan J), pp 113-143. Amsterdam, the Netherlands: Excerpta Medica

7. Crews D (1994) Animal sexuality. Sci Am 270: 109-114

8. Jost A (1947) Sur les effets de la castration precoce de l'embryon male de lapin. CR Seances. Soc Biol Fil 141: 126-129

9. Wallen K, Baum MJ (2002) Masculinization and defeminization in altricial and precocial mammals: Comparative aspects of steroid hormone action. In Hormones, Brain and Behavior (eds Pfaff DW, Arnold AP, Etgen AM, Fahrbach SE, Rubin RT), pp 385-423. San Diego, California, USA: Academic

10. Whalen RE (1974) Sexual differentiation: Models, methods, and mechanisms. In Sex Differences in Behavior (eds Friedman RC, Richart RM, Van de Wiele RL), pp 467-481. New York, New York, USA: Wiley
11. Södersten $P$ (1987) How different are male and female brains? Trends Neurosci 10: 197-198

12. Newman SW (1999) The medial extended amygdala in male reproductive behavior. A node in the mammalian social behavior network. Ann NY Acad Sci 877: 242-257

13. Crews D (2010) Neural control of sexual behaviour. In Encyclopedia of Animal Behavior (eds Breed MD, Moore J), pp 541-548. Oxford, UK: Academic

14. Pfaff DW, Schwartz-Giblin S, McCarthy MM, Kow LM (1994) Cellular and molecular mechanisms of female reproductive behaviors. In The Physiology of Reproduction (eds Knobil E, Neil JD), pp 107-220. New York, New York, USA: Raven

15. Demski LS (1987) Diversity in reproductive patterns and behavior in teleost fishes. In The Psychobiology of Reproductive Behavior: An Evolutionary Perspective (ed Crews D), pp 1-27. Englewood Cliffs, New Jersey, USA: Prentice-Hall

16. Godwin J (2009) Social determination of sex in reef fish. Semin Cell Dev Biol 20: 264-270

17. Rand MS, Crews D (1994) The bisexual brain: sex behaviour differences and sex differences in parthenogenetic and sexual lizards. Brain Res 663: 163-167

18. Kimchi T, Xu J, Dulac C (2007) A functional circuit underlying male sexual behaviour in the female mouse brain. Nature 448: 1009-1014

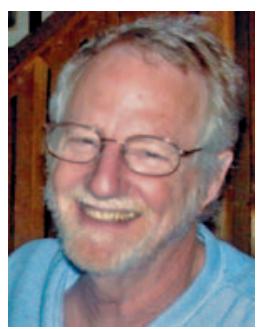

David Crews is at the University of Texas in Austin, Texas, USA.

E-mail:crews@mail.utexas.edu

EMBO reports (2012) 13,779-784; published online 27 July 2012; doi:10.1038/embor.2012.107 